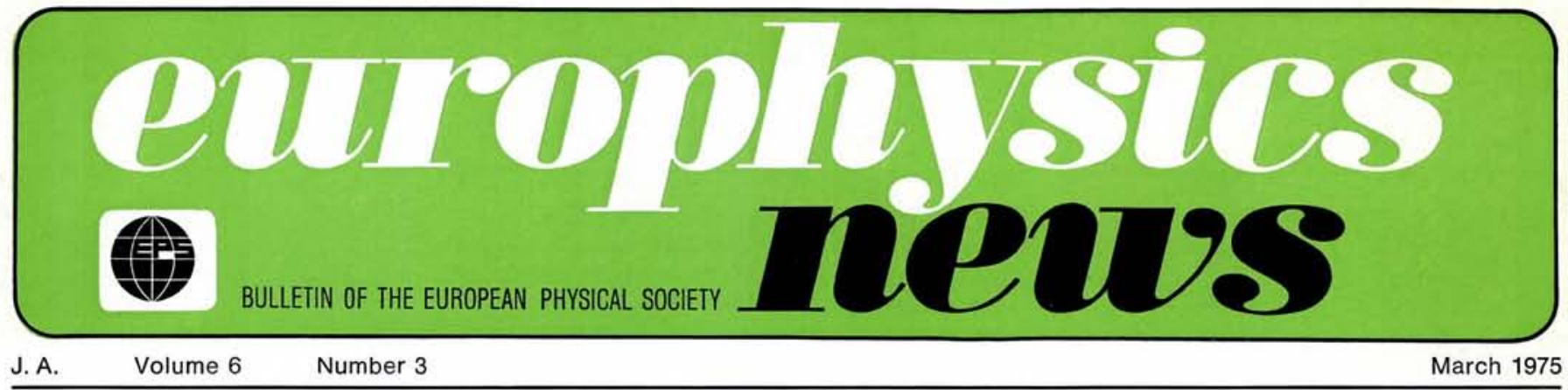

\section{Discovery of Pulsars}

The trail which ultimately led to the first pulsar began in 1948 when I joined Ryle's small research team and became interested in the general problem of the propagation of radiation through irregular transparent media. We are all familiar with the twinkling of visible stars and my task was to understand why radio stars also twinkled. I was fortunate to have been taught by Ratcliffe, who first showed me the power of Fourier techniques in dealing with such diffraction phenomena. By a modest extension of existing theory I was able to show that our radio stars twinkled because of plasma clouds in the ionosphere at heights around $400 \mathrm{~km}$, and I was also able to measure the speed of ionospheric winds in this region ${ }^{1}$ ).

My fascination in using extra-terrestrial radio sources for studying the intervening plasma next brought me to the solar corona. From observations of the angular scattering of radiation passing through the corona, using simple radio interferometers, I was eventually able to trace the solar atmosphere out to one half the radius of the Earth's orbit ${ }^{2}$ ).

In my notebook for 1954 there is a comment that, if radio sources were of small enough angular size, they would illuminate the solar atmosphere with sufficient coherence to produce interference patterns at the Earth which would be detectable as a very rapid fluctuation of intensity. Unfortunately the information then available showed that the few sources known were more than one hundred times too large to produce this effect, and I did not pursue the idea. This was sad because the phenomenon was discovered by chance, about eight years later, by Margaret Clarke long after I had forgotten all about my comment. She was involved with a survey of radio sources at Cambridge and noticed that three particular sources showed variations of intensity. She pointed out that two of the sources were known to have angular Copyright (c) The Nobel Foundation 1975.

\section{Pulsars and High Density Physics}

\section{A. Hewish, Cambridge}

sizes of less than 2" and estimated that a scintillation mechanism required plasma irregularities at distances of thousands of $\mathrm{km}^{3}$ ). During a group discussion I suddenly remembered my earlier conclusion and realised that, if the radio sources subtended an angle of less than 1", they might show the predicted intensity scintillation caused by plasma clouds in the interplanetary medium. With the assistance of Scott and Collins special observations of $3 \mathrm{C} 48$ and other quasistellar radio sources were made and the scintillation phenomenon was immediately confirmed ${ }^{4}$ ).

Since interplanetary scintillation, as we called this new effect, could be detected in any direction in space I used it to study the solar wind, which had by then been discovered by space probes launched into orbits far beyond the magnetosphere. It was interesting to track the interplanetary diffraction patterns as they raced across England at speeds in excess of $300 \mathrm{~km} \mathrm{~s}^{-1}$, and to sample the behaviour of the solar wind far outside the plane of the ecliptic where spacecraft have yet to venture ${ }^{5}$ ).

The scintillation technique also provided an extremely simple and useful means of showing which radio sources had angular sizes in the range 0 ". $1-$ $1 " .0$. The first really unusual source to be uncovered by this method turned up in 1965 when, with my student Okoye, I was studying radio emission from the Crab Nebula. We found a prominent scintillating component within the nebula which was far too small to be explained by conventional synchrotron radiation and we suggested that this might be the remains of the original star which had exploded and which still showed activity in the form of flare-type radio emission $\left.{ }^{6}\right)$. This source later turned out to be none other than the famous Crab Nebula Pulsar.

In 1965 I drew up plans for a radio telescope with which I intended to carry out a large-scale survey of more than 1000 radio galaxies using interplanetary scintillation to provide high angular resolution. To achieve the required sensitivity it was necessary to cover an area of $18,000 \mathrm{~m}^{2}$ and, because scintillation due to plasmas is most pronounced at long wavelengths, I used a wavelength of $3.7 \mathrm{~m}$. The final design was an array containing 2048 dipole antennas. Later that year I was joined by a new graduate student, Jocelyn Bell, and she became responsible for the network of cables connecting the dipoles. The entire system was built with local effort and we relied heavily upon the willing assistance of many members of the Cambridge team.

The radio telescope was complete, and tested, by July 1967 and we immediately commenced a survey of the sky. Our method of utilising scintillation for the quantitative measurement of angular sizes demanded repeated observations so that every source could be studied at many different solar elongations. In fact we surveyed the entire range of accessible sky at intervals of one week.

\section{Contents}

Pulsars and High Density Physics

Activities of the European Science Foundation

5th IAEA Conference on Plasma

Physics and Controlled

Nuclear Fusion Research

Society News

Meetings

1
5
8
8
8




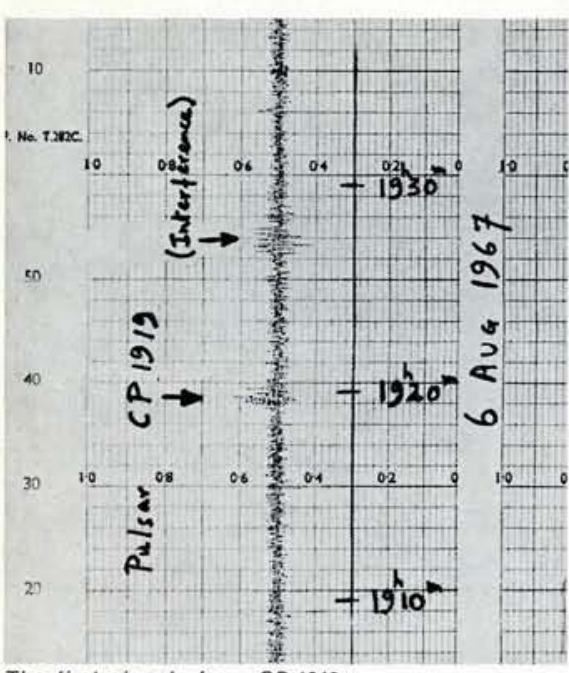

The first signals from CP 1919.

To maintain a continuous assessment of the survey we arranged to plot the positions of scintillating radio sources on a sky-chart, as each record was analysed, and to add points as the observations were repeated at weekly intervals. In this way genuine sources could be distinguished from electrical interference since the latter would be unlikely to recur with the same celestial coordinates. It is greatly to Jocelyn Bell's credit that she was able to keep up with the flow of paper from the four recorders.

One day around the middle of August 1967 Jocelyn showed me a record indicating fluctuating signals that could have been a faint source undergoing scintillation when observed in the antisolar direction. This was unusual since strong scintillation rarely occurs in this direction and we first thought that the signals might be electrical interference. By the end of September the source had been detected on several occasions, although it was not always present, and I suspected that we had located a flare star, perhaps similar to the M-type dwarfs under investigation by Lovell.
We installed a high-speed recorder to study the nature of the fluctuating signals but met with no success as the source intensity faded below our detection limit. During October this recorder was required for pre-arranged observations of another source, $3 \mathrm{C} 273$, to check certain aspects of scintillation theory, and it was not until November 28th that we obtained the first evidence that our mysterious source was emitting regular pulses of radiation at intervals of just greater than one second. I could not believe that any natural source would radiate in this fashion and I immediately consulted astronomical colleagues at other observatories to enquire whether they had any equipment in operation which might possibly generate electrical interference at a fixed sidereal time near $19^{\mathrm{h}} 19^{\mathrm{m}}$.

In early December the source increased in intensity and the pulses were clearly visible above the noise. Still sceptical, I arranged a device to display accurate time marks at one second intervals broadcast from the MSF Rugby time service and on December 11th began daily timing measurements. To my astonishment the readings fell in a regular pattern, to within the observational uncertainty of $0.1 \mathrm{~s}$, showing that the pulsed source kept time to better than 1 part in $10^{6}$. Meanwhile my colleagues Pilkington, and Scott and Collins, found by quite independent methods that the signal exhibited a rapidly sweeping frequency of about $-5 \mathrm{MHz} \mathrm{s}^{-1}$. This showed that the duration of each pulse, at one particular radio frequency, was approximately $16 \mathrm{~ms}$.

Having found no satisfactory terrestrial explanation for the pulses we now began to believe that they could only be generated by some source far beyond the solar system, and the short duration of each pulse suggest-

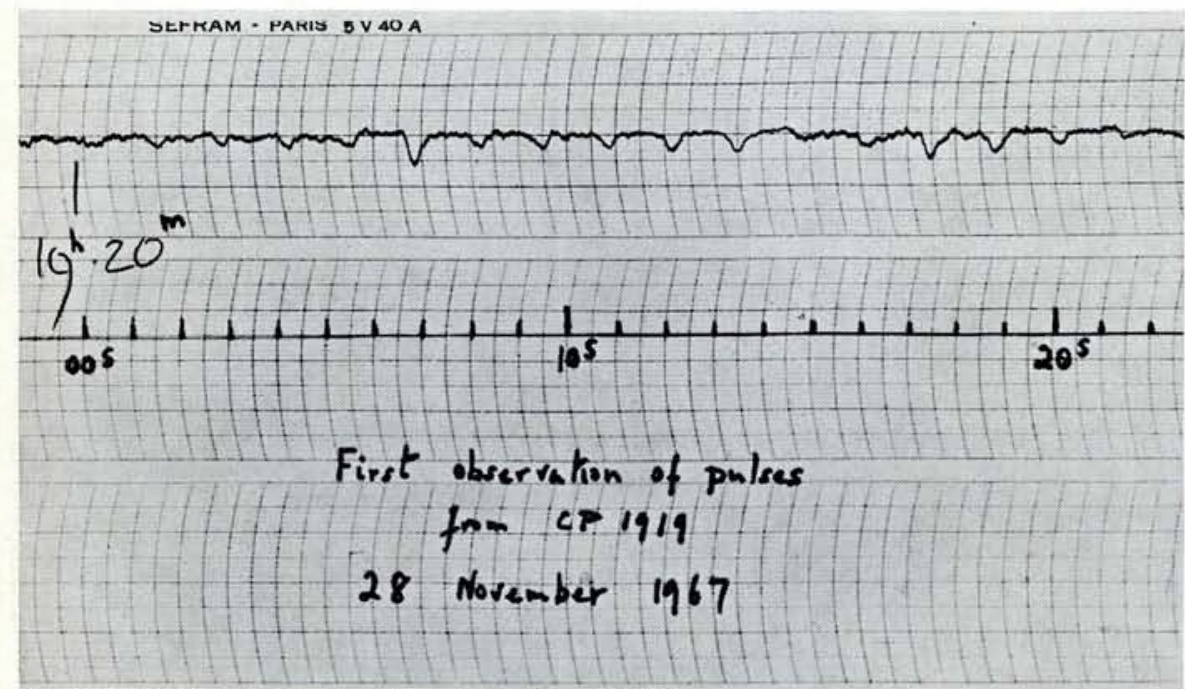

The first indication of pulsed radio emission from CP 1919.

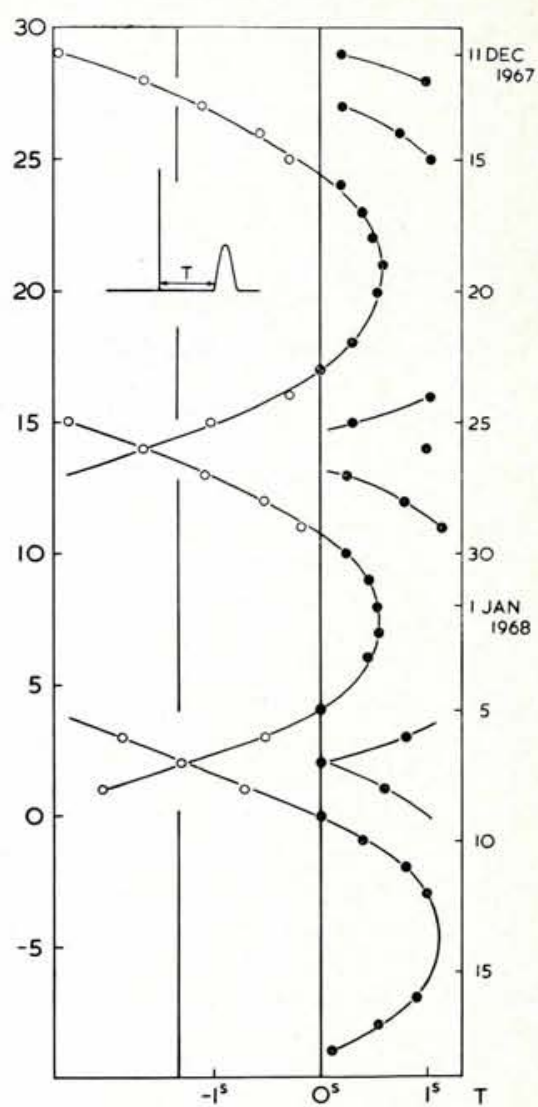

Timing measurements showing Doppler shift due to the orbital motion of the Earth.

ed that the radiator could not be larger than a small planet. We had to face the possibility that the signals were, indeed, generated on a planet circling some distant star, and that they were artificial. I knew that timing measurements, if continued for a few weeks, would reveal any orbital motion of the source as a Doppler shift, and I felt compelled to maintain a curtain of silence until this result was known with some certainty. Without doubt, those weeks in December 1967 were the most exciting in my life.

It turned out that the Doppler shift was precisely that due to the motion of the Earth alone, and we began to seek explanations involving dwarf stars, or the hypothetical neutron stars. My friends in the library at the optical observatory were surprised to see a radio astronomer taking so keen an interest in books on stellar evolution. I finally decided that the gravitational oscillation of an entire star provided a possible mechanism for explaining the periodic emission of radio pulses, and that the fundamental frequency obtainable from white dwarf stars was too low. I suggested that a higher order mode was needed in the case of a white dwarf, or that a neutron star of the lowest allowed density, vibrating in the fundamental mode, might give the required periodicity. We also estimated the distance of the source on the assumption that 


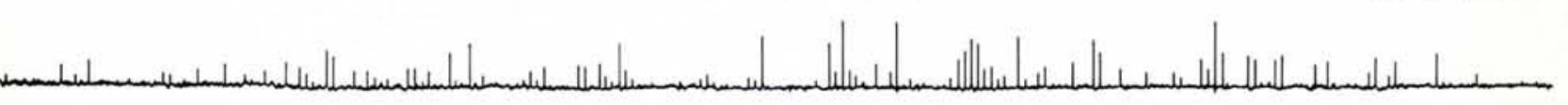

\section{1}

Radiation from a typical pulsar

the frequency sweep was caused by pulse dispersion in the interstellar plasma, and obtained a value of 65 parsec, a typical stellar distance.

While I was preparing a coherent account of this rather hectic research, in January 1968, Jocelyn Bell was scrutinising all our sky-survey recordings with her typical persistence and diligence and she produced a list of possible additional pulsar positions. These were observed again for evidence of pulsed radiation and before submitting our paper for publication, on February 8th, we were confident that three additional pulsars existed although their parameters were then only crudely known. I well remember the morning when Jocelyn came into my room with a recording of a possible pulsar that she had made during the previous night at a right ascension $09^{\mathrm{h}} 50^{\mathrm{m}}$. She initially thought that the fluctuations on the chart were meaningless, but when we spread the chart over the floor and placed a metre rule against it a periodicity of 0.25 s was just discernible. This was confirmed later when the receiver was adjusted to a narrower bandwidth, and the rapidity of this pulsar made explanations involving white dwarf stars increasingly difficult.

The months that followed the announcement ${ }^{7}$ ) of our discovery were busy ones for observers and theoreticians alike, as radio telescopes all over the world turned towards the first pulsars and information flooded in at a phenomenal rate. It was Gold ${ }^{8}$ ) who first suggested that the rotation of neutron stars provided the simplest and most flexible mechanism to explain the pulsar clock, and his prediction that the pulse period should increase with time soon received dramatic confirmation with the discovery of the pulsar in the Crab Nebula ${ }^{9}{ }^{10}$ ). Further impressive support for the neutron star hypothesis was the detection of pulsed light from the star which had previously been identified as the remnant of the original explosion. This, according to theories of stellar evolution, is precisely where a young neutron star should be created. Gold also showed that the loss of rotational energy, calculated from the increase of period for a neutron star model, was exactly that needed to create the observed synchrotron light from the nebula.

Now, in 1974, with more than 130 pulsars charted in the heavens, there is overwhelming evidence that the neutron star "lighthouse" model is correct. No other star could spin fast enough, without fragmenting, to account for the most rapid pulsars yet periods ranging from $33 \mathrm{~ms}$ to $3.5 \mathrm{~s}$ are readily accommodated by the rotation theory. At the same time there is unfortunately no satisfactory theory to account for the radio emission generated by these tiny stars which have radii of only $10 \mathrm{~km}$.

\section{High Density Physics inside Neutron Stars}

The prediction that matter at the almost unimaginable density of $10^{18} \mathrm{~kg} \mathrm{~m}^{-3}$ might be formed under gravitational compression inside stars was first made by Landau ${ }^{11}$ ) in 1932, soon after Chadwick's discovery of the neutron. At this density only a small fraction of the original protons and electrons could exist and matter would consist predominantly of neutrons. It is the degeneracy pressure arising from the neutrons, which obey Fermi statistics, that balances further gravitational compression, although finally the Fermi energy becomes relativistic and further gravitational collapse ensues. Since complex nuclei are generated by nuclear fusion inside hot stars, where there is a large thermal pressure, the degenerate neutron state can only be found when fusion ceases and we deal with the cooling "ashes" of stellar evolution. The stars that give rise to neutron stars are more massive than the Sun, and it is believed that the formation of neutron stars is associated with supernova explosions.

Since the discovery of pulsars there has been great activity amongst solidstate physicists around the world because neutron matter, at any temperature less than about $10^{\circ} \mathrm{K}$, behaves rather like ordinary matter close to the absolute zero of temperature. The generally agreed model of a neutron star consists of concentric shells with very different physical properties as reviewed by Ruderman ${ }^{12}$ ).
At the surface of the star it is likely that there exists a shell of iron since ${ }^{56} \mathrm{Fe}$ is the most stable nucleus. The atoms would be normal if no magnetic field were present. In astrophysics it is unwise to ignore magnetic phenomena and gravitational collapse following a supernova explosion probably compresses the original stellar magnetic flux to produce surface field strengths of $10^{8} \mathrm{~T}$ or more. In fields of this magnitude the radius of gyration of electrons in atomic energy levels becomes smaller than the Bohr radius and the electronic wave functions adopt a cylindrical shape. It is far harder to ionize distorted atoms of this kind and this is of importance when considering the generation of a magnetosphere surrounding the neutron star.

Beneath the iron skin the increasing compression forces electrons into higher energy states until they are entirely freed from the positive nuclei. The unscreened nuclei then settle into a rigid lattice having a melting temperature of about $10^{\circ} \mathrm{K}$. At greater depths the electron energies become relativistic and they begin to combine with protons in the nuclei, thus adding to the neutron population. This is the process of inverse $\beta$ decay. At a sufficient depth nearly all the electrons and protons have disappeared and the nuclei have been converted to a sea of neutrons.

The energy gap for neutron pairing is of the order of several MeV, corresponding to a superfluid transition temperature of $10^{9}-10^{10} \mathrm{~K}$, and since young neutron stars cool rapidly to temperatures below $10^{\circ} \mathrm{K}$, the neutron sea is expected to behave like a quantum superfluid. The few remaining protons will similarly pair and enter a superconducting state, while the residual electrons will behave normally. The bulk motion of the neutron superfluid must be irrotational, but an effective solid body rotation can be simulated with a distribution of quantised vortex lines containing a small fraction of normal fluid neutrons.

At yet deeper levels the neutronneutron interaction may result in the creation of a solid neutron lattice, although this possibility is under debate, and finally there is the question 


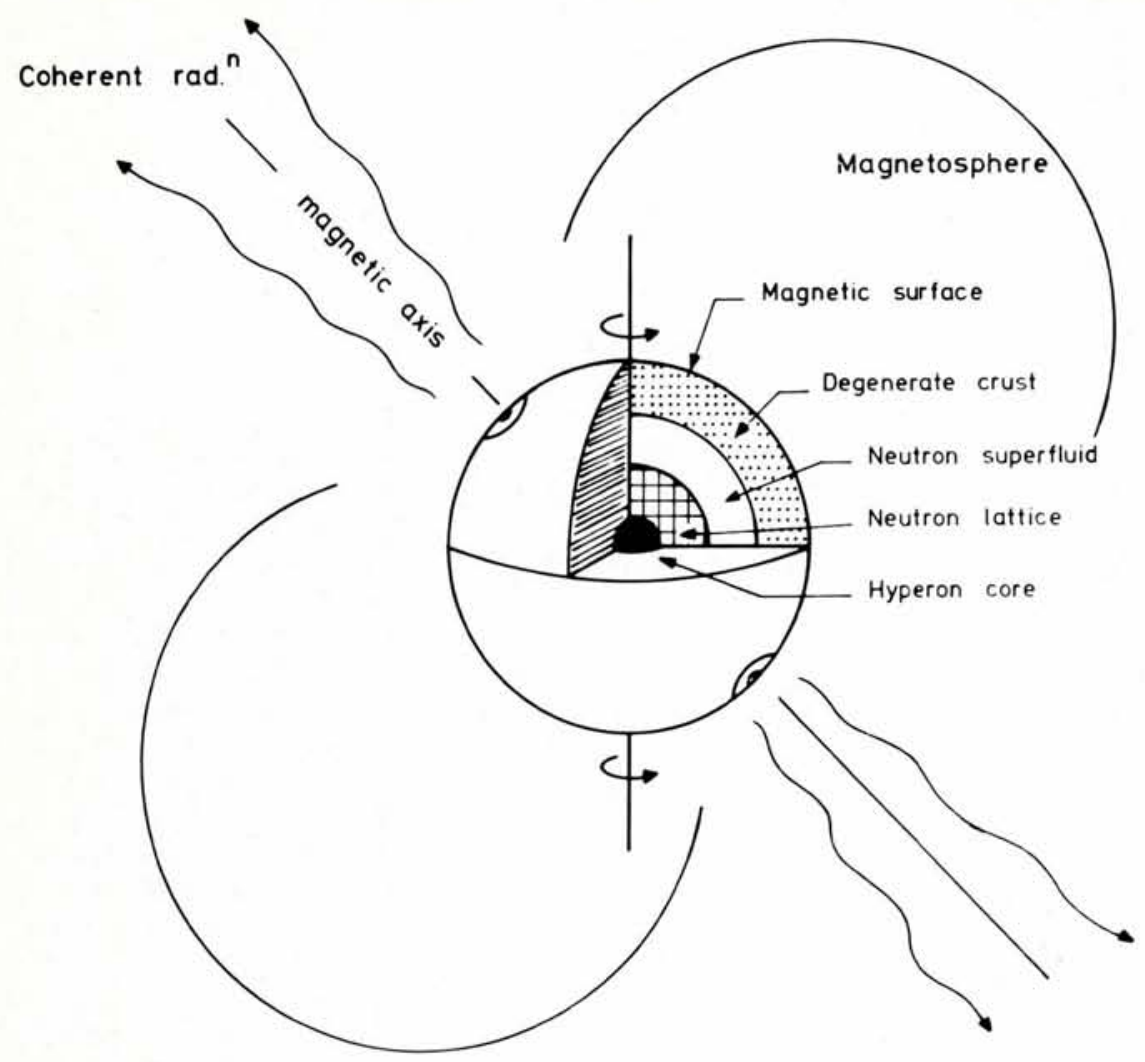

Model of a neutron star.

of a material composed of stable hyperons.

Evidence that neutron stars do indeed have a structure similar to the predicted models has been obtained from extended timing observations of pulsars. These show that the systematic increase of period, corresponding to a steady loss of rotational energy from the spinning star, is sometimes interrupted by discontinuous changes. Most pulsars are observed to be slowing down on a typical timescale of $10^{6}-10^{7}$ years, although the most rapid pulsars, in the Crab and Vela supernovae, have timescales of only $10^{3}$ and $10^{4}$ years respectively. The discontinuities often show an abrupt decrease of period, followed by a recovery to a slightly reduced value with a characteristic relaxation time.

For the Crab pulsar this effect can be explained by a rigid crust-liquid core model. Young neutron stars are likely to be spinning rapidly at birth, with angular velocities up to $10^{4}$ radian $\mathrm{s}^{-1}$, and they will therefore have a spheroidal shape. As a star slows down it will tend to become less spheroidal and the rigid crust will fracture at irregular intervals as the increasing strain overcomes rigidity. When this occurs the crust will momentarily spin more rapidly, but later the increased angular momentum will be coupled into the fluid interior, where the bulk of the mass resides. The observed time constant for coupling is in good agreement with the
It is strange that there appears to be more understanding of the interior of neutron stars, than of their atmospheres wherein is generated the radiation which makes them detectable. Ginzburg and Zheleznyakov ${ }^{13}$ ) have summarised the electrodynamic problems in detail. The model upon which theorists are concentrating most attention is that of an oblique magnetic rotator, in which the pulsar may be regarded as a dynamo, powered by the initial store of rotational kinetic energy, and converting this into radiation together with a flux or relativistic particles by means of the large magnetic field. The oblique rotator model was first considered by Pacini ${ }^{14}$ ) before pulsars had been found, and it was Gold ${ }^{8}$ ) who suggested that an extended corotating magnetosphere played a vital role.

Goldreich and Julian ${ }^{15}$ ) showed that electrical forces arising from unipolar induction would be sufficient to drag charges from the stellar surface and then distribute them in a corotating magnetosphere. It is not yet known whether such a distribution is stable, and the plasma differs from laboratory plasmas in that almost complete charge separation occurs. Inertial forces must dominate when the corotation velocity approaches $\mathrm{c}$, and beyond the velocity of light cylinder the plasma breaks away to create a stellar wind. In such models the polar regions are believed to play a crucial role since particles can escape along 'open' field lines.

Within such an overall framework exists the ordered motion of the charges which generate the beamed radio waves that we observe, and also those regions which emit light and $\mathrm{X}$-rays for the youngest pulsar in the Crab. The fascinating richness of the phenomena involving polarisation,

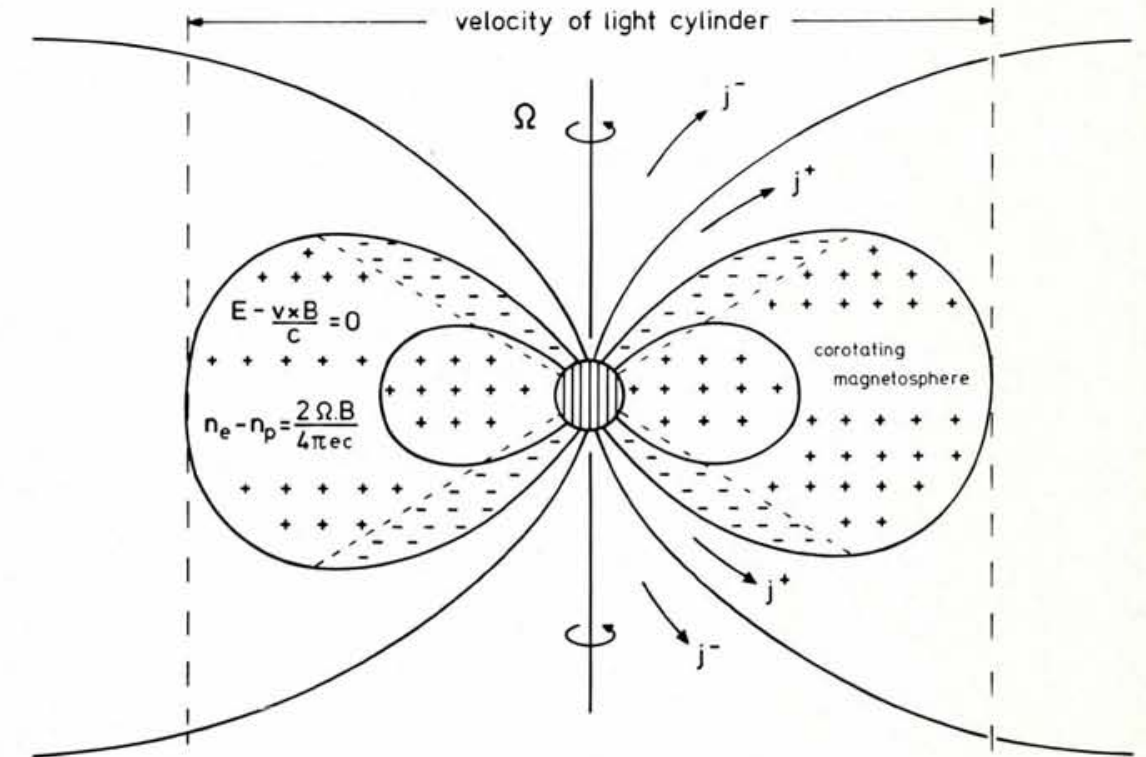

The neutron star magnetosphere for an aligned magnetic rotator. 
pulse shapes, radio spectra, intensity variations, and complex secondary periodicities, must eventually provide vital evidence to resolve our present uncertainties. There is good reason to believe that the general outline is correct. Simple dynamics shows that the surface magnetic field strength $B_{\circ}^{2}$ is proportional to $P \frac{d P}{d t}$, where $P$ is the pulsar period, and observations of many pulsar give $B_{\circ} \sim 10^{8} \mathrm{~T}$ when conventional neutron star models are assumed. Further evidence comes from pulsar ages which are approximately $P\left(\frac{d P}{d t}\right)^{-1}$. Typical ages are $10^{6}-10^{7}$ years although $10^{3}$ years is obtained for the Crab pulsar, in good agreement with the known age of the supernova.

\section{Conclusion}

In outlining the physics of neutron stars, and my good fortune in stumbling upon them, I hope that I have given some idea of the interest and rewards of extending physics beyond the confines of laboratories. These are good times in which to be an astrophysicist. I am also deeply aware of my debt to all my colleagues in the Cavendish Laboratory. Firstly to Sir Martin Ryle for his unique flair in creating so congenial and stimulating a team in which to work. Secondly to Jocelyn Bell for the care, diligence and persistence that led to our discovery so early in the scintillation programme, and finally to my friends who contributed so generously in many aspects of the work.

\section{REFERENCES}

1) HEWISH, A., Proc. Roy. Soc. (London) 214 (1952) 494.

2) HEWISH, A., and WYNDHAM, J.D., Mon. Not. R. astr. Soc. 126 (1963) 469

3) CLARKE, M.E., Ph. D. Thesis. Cambridge (1964).

4) HEWISH, A., SCOTT, P.F., and WILLS, D., Nature 203 (1964) 1214.

5) DENNISON, P.A., and HEWISH, A., Nature 213 (1967) 343.

6) HEWISH, A., and OKOYE, S.E., Nature 207 (1960) 59 .

7) HEWISH, A., BELL, S.J., PILKINGTON, J.D.H., (1968) 709.

8) GOLD, T., Nature 218 (1968) 731.

9) STAELIN, D.H., and REIFENSTEIN, E.C. Science 162 (1968) 1481.

10) COMELLA, J.M., CRAFT, H.D., LOVELACE R.V.E., SUTTON, J.M., and TYLER, G.L., Nature 221 (1969) 453.

11) LANDAU, L., Phys. Zeits. Sowjetunion 1 (1932) 285.

12) RUDERMAN, M., Ann. Rev. Astron. Astrophys. 10 (1972) 427.

13) GINZBURG, V.L., and ZHELEZNYAKOV, V.V., Ann. Rev. Astron. Astrophys. 13 (1975) in press.

14) PACINI, F., Nature 219 (1968) 145.

15) GOLDREICH, P., and JULIAN, W.H., Astrophys. J. 157 '(1969) 869.

\section{Activities of the European Science Foundation}

\section{E. N. Shaw, Geneva}

The absence of any clear-cut programme of work or even an explicit set of immediate objectives obscures the fact that already some years of hard work lie behind the formation of the European Science Foundation (ESF). Two separate initiatives have come together in the process, one starting off in the Council of Ministers of the EEC which instructed the Commission to consider how to coordinate pure research in the common market countries and the other in Scandinavia and the UK which aimed at a closer contact between science research councils in Western European States.

It is to the credit of both groups and to the advantage of all that what has emerged is a broadly based, openended society, covering 16 nations with unrestrictive statutes and motivated by a determination to get something done. The countries represented are as follows : Austria, Belgium, Denmark, France, Fed. Rep. of Germany, Greece, Ireland, Italy, Netherlands, Norway, Portugal, Spain, Sweden, Switzerland, Yugoslavia, United Kingdom.

Following the first plenary meeting of the Foundation in Strasbourg on November 18 and 19, the newly elected president, Sir Brian Flowers, emphasised that the immediate problem was not to find suitable subjects, but to establish priorities amongst the plethora of possibilities which presented themselves.

Science in the context of the ESF, it should be stressed, is not confined to the "hard sciences" but extends firmly into the humanities, jurisprudence, social sciences and economics underligned by the fact that of the two vice-presidents, Professor $\mathrm{O}$. Reverdin, president of the Swiss National Scientific Research Foundation, is described as a hellenist and Doctor $\mathrm{P}$. Riis is a medical doctor in Copenhagen.

One of the first questions on the Foundation's agenda was the future of the West European Science Council which had been formed in 1972 and the European Medical Research Council which has also been in existence for some time. Although there is clearly a real overlap with the work of the ESF it was felt that both these two organizations should be continued and a form of association found which would avoid duplication of effort.

\section{Membership}

Membership of the Foundation is restricted to research councils and academies which have substantial government backing. The Foundation is not directly sponsored by governments but is only one step away. The idea is to bring together from the participating countries, in one assembly, influential men from science administration and the "elder statesman" of science.

The number of member organizations is not limited and varies according to the infrastructure of the States. Representatives of these bodies make up the Assembly out of which is formed the Executive Council of 18 members, comprising at least one member from each of the countries involved.

In the choice of the president and vice presidents, it is not accidental that the UK, Denmark and Switzerland are represented while the secretarygeneral is Dr. F. Schneider of the Max-Planck Institute at Munich (Fed. Rep. of Germany) and the offices of the organization will be in Strasbourg (France). It is considered important to maintain a wide geographical representation as well as a wide subject coverage in the governing bodies.

Tribute was paid to the efforts of Dr. Dahrendahl former commissioner for science and research of the European Communities and also to the founding committee under Professor $\mathrm{H}$. Curien whose proposals were formally ratified at the plenary meeting.

\section{Statutes}

In the statutes, the principal objects are stated as :

a) to advance cooperation in basic research;

b) to promote mobility of research workers:

c) to assist the free flow of ideas and information ;

\section{The UNIVERSITY of GENEVA \\ is opening an inscription for a position of}

Professor of Theoretical Physics

Candidature must be sent before April 30, 1975, to the Secretariat of the Faculty of Sciences, 20 quai Ernest Ansermet, 1211 Geneva 4, where additionnal information may be obtained. 